\title{
Decrease of postprandial endothelial dysfunction by spice mix added to high-fat hamburger meat in men with Type 2 diabetes mellitus
}

\author{
Z. Li ${ }^{1}$, S. M. Henning ${ }^{1}$, Y. Zhang ${ }^{1}$, N. Rahnama ${ }^{1}$, A. Zerlin ${ }^{1}$, G. Thames ${ }^{1}$, C. H. Tseng ${ }^{2}$, D. \\ Heber $^{1}$ \\ ${ }^{1}$ Center for Human Nutrition, David Geffen School of Medicine, University of California, Los \\ Angeles, CA, USA \\ ${ }^{2}$ Statistics Core, Department of Medicine, David Geffen School of Medicine, University of \\ California, Los Angeles, CA, USA
}

\begin{abstract}
Aims-Consumption of a high-fat diet has been demonstrated to promote endothelial dysfunction, possibly through an increase in lipid peroxidation and decrease in serum nitric oxide. The present study was designed to investigate whether consumption of a hamburger cooked with a polyphenol-rich spice mixture will reduce postprandial lipid oxidation and endothelial dysfunction in men with Type 2 diabetes.
\end{abstract}

\begin{abstract}
Methods-Twenty-two subjects consumed burgers cooked with salt only (control burger) or with salt and spice mix (spice burger) in randomized order. The postprandial concentration of urinary malondialdehyde and nitrate/nitrite as well as the peripheral arterial tonometry score were determined.
\end{abstract}

Results-Eighteen subjects completed the study. Postprandial serum glucose, insulin and triglyceride concentrations were similar in all subjects after control burger or spice burger consumption. Urine malondialdehyde excretion in mmol/g creatinine was reduced by $31 \%$ ( $P<$ 0.001 ) after consuming the spice burger compared with the control burger. Two hours after consumption of the burgers, the peripheral arterial tonometry score was significantly different between control burger consumption $(-9.7 \pm 21.5 \%)$ and spice burger consumption $(+18.0 \pm$ 42.4\%) $(P=0.025)$. Mean urinary nitrate/nitrite concentrations in urine collected during the $6 \mathrm{~h}$ after consumption of the control burger was $9.09 \pm 5.7 \mathrm{mmol} / \mathrm{g}$ creatinine, but $12.37 \pm 7.00$ $\mathrm{mmol} / \mathrm{g}$ creatinine after the spice burger $(P=0.053)$.

Conclusion-Adding a spice mix to hamburger meat prior to cooking resulted in a reduction in urinary malondialdehyde, an increase in urinary nitrate/nitrite and improvement of postprandial endothelial dysfunction in men with Type 2 diabetes. Therefore, cooking a hamburger with a polyphenol-rich spice mixture may lead to potential cardiovascular benefits in patients with Type 2 diabetes mellitus.

Correspondence to: David Heber. dheber@mednet.ucla.edu.

Competing interests

None declared. 


\section{Introduction}

Polyphenols are the largest group of secondary metabolites produced by plants, mainly, in response to biotic or abiotic stresses such as infections, injuries, ultraviolet irradiation, exposure to ozone, pollutants and other hostile environmental conditions. These phenolic compounds are one of the main class of biologically active components of spices, aromas, essential oils and traditional medicines [1]. Among commonly eaten foods, spices have the highest known concentrations of antioxidant and anti-inflammatory compounds.

Over the past 20 years, the pathophysiological role of oxidized lipids, in the development of atherosclerosis, has been demonstrated in both basic and clinical studies [2]. The use of antioxidants from dietary sources, including herbs and spices, to prevent lipid oxidation has been proposed as an adjunct to other preventive measures, such as achieving and maintaining a healthy body weight and controlling blood cholesterol levels. In addition to their antioxidant activity, polyphenols may also contribute to cardiovascular health by improving the endothelial control of vascular tone. For example, spices have been shown to induce nitric oxide-mediated endothelium-dependent relaxation of isolated arteries [3]. Recently, we reported that the production of malondialdehyde in burgers was reduced by $71 \%$ when a spice mix was added prior to cooking. We also demonstrated a decrease in plasma and urinary malondialdehyde in healthy study participants consuming the burgers prepared with a spice mix compared with burger prepared with salt only [4].

High-calorie meals rich in saturated fat can lead to transient elevations in serum triglycerides and insulin [5]. This condition, termed postprandial dysmetabolism, generates excess free radicals (or reactive oxygen species). The ensuing oxidative stress triggers a biochemical cascade throughout the circulation, inducing inflammation and endothelial dysfunction. These postprandial changes, when repeated multiple times each day, can create a milieu conducive to the development of atherosclerotic risk factors and coronary heart disease, the main cause of morbidity and mortality in the Western world. Several hypotheses have been articulated to explain the initiating events in atherosclerosis, but its pathogenesis seems to be multifactorial, including effects of postprandial oxidized lipids [6-9].

Type 2 diabetes mellitus is a major risk factor for atherosclerosis and it is known that a highfat meal can induce both oxidant and inflammatory stress [10,11]. Patients with Type 2 diabetes exhibit exaggerated and prolonged postprandial lipidaemia [12]. It has been shown that fatty meals induce decreases in endothelial function and oxidative stress is greater in subjects with Type 2 diabetes than healthy controls [12]. The present study was designed to investigate whether spice polyphenols reduce the intestinal and systemic formation of cytotoxic lipid peroxidation products and increase nitric oxide formation, thereby leading to improved endothelial vascular function in men with Type 2 diabetes.

\section{Subjects and methods}

The study protocol was approved by the University of California Los Angeles (UCLA) Institutional Review Board. All subjects gave written informed consent prior to any study procedures being conducted. The study utilized a randomized crossover design. Subjects 
consumed, in a random order, two different test meals consisting of either: (1) ground beef seasoned with salt, or (2) ground beef seasoned with the spice mixture. The test meal and spice mix used are the same as described previously [4]; i.e. $11.25 \mathrm{~g}$ spice mix (cloves $4 \%$, cinnamon $4 \%$, oregano $26 \%$, rosemary $4 \%$, ginger $11 \%$, black pepper $7 \%$, paprika $30 \%$ and garlic powder 13\%) and $2.5 \mathrm{~g}$ of salt were mixed with $250 \mathrm{~g}$ ground beef (10\% fat).

\section{Study participants}

Twenty-two participants were recruited to the study with the following characteristics: 35 70 years of age, a diagnosis of Type 2 diabetes being treated with oral agents, $\mathrm{HbA}_{1 \mathrm{c}}$ level $\leq$ $9.0 \%$ at screening, or with a diagnosis of impaired glucose tolerance on no medications with a fasting blood sugar $>6.1 \mathrm{~mol} / \mathrm{l}$, but less than $7.0 \mathrm{mmol} / \mathrm{l}$ and BMI $>25 \mathrm{~kg} / \mathrm{m}^{2}$. Candidates were excluded if they were taking dietary supplements, smoked $>1$ cigarette/day, exercised heavily ( $>4 \times 30 \mathrm{~min}$ of aerobic exercise/week), drank more than two glasses of wine, two cocktails or two beers/day, or were taking anti-inflammatory medications and steroids. Eighteen participants finished the intervention. Concomitant medication is described in Table 2.

\section{Study protocol}

Eligible participants were asked to avoid eating meat, poultry or fish products for 3 days before the day of each of the two experimental visits. Participants arrived at the UCLA Center for Human Nutrition after fasting for $10 \mathrm{~h}$. Fasting blood glucose was tested and only the participants with blood glucose between 5 and $11.1 \mathrm{mmol} / \mathrm{l}$ proceeded. Participants with fasting glucose out of this range were rescheduled and excluded if the repeat visit still had an out-of-range fasting glucose level. On the intervention day, after an indwelling catheter was inserted into the vein of the forearm and a blood sample was taken, the first measurement of peripheral arterial tonometry was performed. The study participants either consumed the control or spice burger in a randomized order on two separate days 1 week apart.

Participants were asked to finish eating within $30 \mathrm{~min}$. For subjects who routinely took metformin in the morning, the usual dose of metformin was given just before the consumption of the test hamburger and all other diabetes medication were withheld on the test day.

Blood samples were drawn again at 1, 2, 3, 4, 5 and $6 \mathrm{~h}$ after the meal for insulin, glucose, triglyceride and nitrate/nitrite analyses. Peripheral arterial tonometry was repeated after $2 \mathrm{~h}$. Participants were recruited at two different time periods. Only for participants in the second group, urine was collected in a 2-1 container for $6 \mathrm{~h}$ after the meal. Urine volume was recorded and aliquots were taken for analysis of malondialdehyde and creatinine.

\section{Biochemical analysis}

Ethylene-diaminetetraacetic acid (EDTA) plasma and serum were separated from whole blood by centrifugation $(910 \mathrm{~g}, 15 \mathrm{~min})$, frozen and kept at $-80{ }^{\circ} \mathrm{C}$ pending malondialdehyde and nitrate/nitrite determination ( $<1$ week). Serum insulin concentration was determined using the Siemens Immulite Chemiluminescence Immunoassay System (Siemens Healthcare Diagnostics, Dallas, TX, USA). This assay is a solid-phase, two-site chemiluminescent immunometric assay using beads coated with monoclonal murine anti- 
insulin antibody. Interassay coefficient of variation for insulin: 5.9\% (0.29 pmol/l), 7.0\% (3.4 pmol/l). Serum glucose was determined using the Cayman Glucose Assay kit (Cayman Chemical Company, Ann Arbor, MI, USA) based on a colorimetric determination using the glucose oxidase-peroxide reaction. Serum triglyceride concentration was determined using standard enzymatic methods. Reagents, standards and calibrators were purchased from Pointe Scientific (Lincoln Park, MI, USA). The interassay coefficients of variation are less than $4 \%$ and intra-assay variation is less than $2 \%$.

Urine content of malondialdehyde was analysed by alkaline hydrolysis, acid deproteinization, derivatization with tetrabutylammonium (TBA) and $n$-butanol extraction following the method of Grotto et al. [13] and described in detail previously [4].

\section{Peripheral arterial tonometry}

According to previous studies [14], a blood pressure cuff was placed on one upper arm (study arm), while the other arm served as a control (control arm). Peripheral arterial tonometry probes were placed on one finger of each hand for continuous recording of the peripheral arterial tonometry signal. After a 10-min equilibration period, the blood pressure cuff was inflated to suprasystolic pressures for $5 \mathrm{~min}$. Then the cuff was deflated, while peripheral arterial tonometry recording was continued for $10 \mathrm{~min}$.

The reactive hyperemia peripheral arterial tonometry (RH-PAT) data were analysed by a computer in an operator-independent manner as previously described [15]. As a measure of reactive hyperemia, the reactive hyperemia peripheral arterial tonometry index was calculated as the ratio of the average amplitude of the peripheral arterial tonometry signal over a 1-min time interval starting $1 \mathrm{~min}$ after cuff deflation divided by the average amplitude of the peripheral arterial tonometry signal of a 3.5-min time period before cuff inflation (baseline). Subsequently, reactive hyperemia peripheral arterial tonometry index values from the study arm were normalized to the control arm.

\section{Nitrate/nitrite determination in urine}

Nitric oxide is oxidized in the body to the metabolites nitrate $\left(\mathrm{NO}^{-}\right)$and nitrite $\left(\mathrm{NO}^{-}\right)$, which are excreted in the urine. In the urinary sample, the sum of nitrate/nitrite was measured as an indirect indicator of the nitric oxide production. The urinary nitrate/nitrite level was determined by using the DetectX Nitric Oxide Detection Kit (ArborAssay, Ann Arbor, MI, USA) according to manufacturer instructions. Briefly, both nitrate and nitrite standards calibration curves were generated by mixing with the colour reagents $\mathrm{A}$ and $\mathrm{B}$ provided by the kit and incubated at room temperature $\left(22^{\circ} \mathrm{C}\right)$ for $5 \mathrm{~min}$. Following a 20min incubation with nitrate reductase and nicotinamide adenine dinucleotide (NADH) total urinary nitrate/nitrite content was measured. The reductase in combination with NADH reduced nitrate to nitrite. After another 5-min incubation with colour reagents A and B at room temperature, the concentration of the coloured product was read at $570 \mathrm{~nm}$.

The urinary creatinine level was measured by using a Stanbio Direct Creatinine LiquiColor Kit (Stanbio Laboratory, Boerne, TX, USA). A creatinine standard calibration curve was generated by plotting the absorbance measured at $510 \mathrm{~nm}$ of a series concentration of creatinine standard solutions reacting with working reagent provided by the measuring kit. 
The urinary creatinine level was measured in the same manner after a proper dilution with distilled water.

\section{Statistics}

Data were expressed as mean \pm standard error of means or standard deviation. A logtransformation on the value of thiobarbituric acid reactive substances was applied to satisfy the normality assumption of the model. A linear mixed-effects model to evaluate the effect of meal type on the production of thiobarbituric acid reactive substances was utilized. The fixed effects in the model included meal type, time and meal type/time interaction. A random intercept was used to accommodate the correlation from the same subject. After imputation, we calculated the ratio of thiobarbituric acid reactive substances at each time point to its baseline value. The primary statistical analysis tool was the generalized estimating equations method to analyse the repeated measurements of plasma malondialdehyde for its robust statistical inference. Generalized estimating equations was used to analyse the repeated ratios of each subject with an unstructured working covariance matrix. Differences were considered significant at $P<0.05$. The SAS 9.1.3 software package (SAS Institute, Cary, NC, USA) was used.

\section{Results}

Thirty-nine subjects were screened and 22 subjects met both the inclusion and exclusion criteria and were enrolled into the study. The demographics of all enrolled subjects are shown in Table 1. One subject was eliminated because of dietary non-compliance, with a repeated fasting blood glucose $>200 \mathrm{mg} / \mathrm{dl}$. Three subjects did not complete the study visits within the study window because of conflicting schedules and were excluded. Thirteen out of 18 completed subjects were taking metformin on the morning of testing. The burgers and study protocol were tolerated well and none of the subjects had any adverse event.

There was no significant difference in the postprandial concentration of glucose, insulin and triglycerides in blood from participants consuming the control or spice burger (Fig. 1). Changes over time were not significant. Urine malondialdehyde excretion in $\mu \mathrm{mol} / \mathrm{g}$ creatinine was reduced by $31 \%(P<0.001)$ in the participants consuming the spice burger compared with those in the group consuming the control burger (Fig. 2).

The baseline peripheral arterial tonometry score was $1.8 \pm 0.6$ in the participants consuming the control burger and $1.7 \pm 0.4$ in those consuming the spice burger. Two hours after the consumption of the test burger, the score decreased by $9.7 \pm 21.5 \%$ for participants consuming the control burger and increased by $18.0 \pm 42.4 \%$ for participants consuming the spice burger. The change of peripheral arterial tonometry score after control burger and spice burger consumption was significantly different $(P=0.025)$ (Fig. 3).

The average urine nitrate/nitrite concentration $6 \mathrm{~h}$ after consumption of the burgers was 9.09 $\pm 5.7 \mathrm{mmol} / \mathrm{g}$ creatinine after the control burger and $12.37 \pm 7.00 \mathrm{mmol} / \mathrm{g}$ creatinine after the spice burger consumption, with a $P$-value of 0.053 (Fig. 4). 


\section{Discussion}

The results of the present study demonstrated that the consumption of a high-fat meal was associated with postprandial vasoconstriction. We were able to demonstrate for the first time that the addition of a spice mix high in antioxidant polyphenols to a high-fat meal led to a significant increase in postprandial vascular dilation in men with Type 2 diabetes. This vasodilation was associated with an increase in urinary nitrate/nitrite.

The beneficial effects of polyphenols on cardiovascular disease have been attributed to their ability to reduce vascular oxidative stress. Polyphenols are able to reduce oxidative stress through their free radical scavenging activity, their stimulatory effect on endogenous antioxidant enzymes and inhibitory effect on pro-oxidant enzymes [16,17]. As demonstrated in our previous hamburger spice study, the addition of the antioxidant spice mix to the burger prior to cooking reduced the formation of malondialdehyde by 60\% [4]. As reviewed by Kanner et al. additional lipid peroxidation is taking place in the stomach. The addition of red wine polyphenols to the meat after cooking, prior to consumption, decreased the lipid peroxidation by $75 \%$ [18]. This demonstrates that the antioxidant polyphenols provide protection during cooking and during the digestion process. In the present study, we found that the addition of spice polyphenols to a hamburger meal was associated with a decrease in urinary malondialdehyde. Possible mechanisms are a decrease in the formation of lipid peroxides in the stomach or increase in the formation of an adduct between polyphenols and aldehydes, such as found between polyphenols and methyl glyoxal [19,20]. Lo et al. demonstrated similar interactions between tea catechins and methyl glyoxal [19].

Polyphenols may also react with proteolytic enzymes in the gastrointestinal tract. Digestion of foods with proteolytic enzymes in vitro releases malondialdehyde from proteins, mainly in the form of adducts with lysine [21]. In animals, malondialdehyde-lysine is found in the liver, small intestine and plasma [22]. Secondary bonds formed between polyphenols and proteins [23] or enzymes [24,25] may also prevent digestion of the malondialdehyde-protein adduct to form malondialdehyde-lysine in the gut and so decrease malondialdehyde absorption. Another benefit of consuming spice polyphenols as an integral part of meals may be their ability to prevent generation and absorption of cytotoxic advanced lipid peroxidation end products [26], such as reactive carbonyls or other reactive compounds commonly found in high-fat foods. Diets high in fat and red meat are risk factors for cardiovascular disease. Locating the biological site of action of polyphenols in the gastrointestinal tract may lead to a revision in our understanding of how antioxidants work in vivo and may help to elucidate the mechanism involved in the reduced risk of heart disease observed with diets rich in antioxidants.

In addition to the antioxidant effect, both experimental and clinical studies indicate that polyphenols may also protect the cardiovascular system by improving the endothelial function. The endothelium plays a major role in the control of the vascular tone through release of nitric oxide (NO). Conditions contributing to diabetic vascular remodelling and dysfunction include the effects of oxidative stress and decreased nitric oxide bioavailability. Nitric oxide production by endothelial nitric oxide synthase (eNOS) is critically involved in maintaining the integrity and stability of the vascular endothelium. Overproduction of superoxide can lead to scavenging of nitric oxide and to its reduced bioavailability [27]. 
Changes in the endothelium attributable to Type 2 diabetes may be associated with abnormalities of vascular tone and helps to detect changes using peripheral arterial tonometry in our study patients compared with healthy volunteers. As found in the present study, in Type 2 diabetes, the urinary nitrate/nitrite concentration showed a strong trend $(P=$ $0.053)$ to be increased in men consuming the spice burger. It has been demonstrated that polyphenols induce nitric oxide formation through the redox-sensitive activation of the phosphatidylinostiol-3-kinase (PI3K)/Akt pathway and by increasing the intracellular calcium concentration leading to the activation of endothelial nitric oxide synthase [28]. The increase in plasma levels of nitroso adducts and enhanced bioavailability of nitric oxide has also been demonstrated in human studies of consumption of high-flavanol cocoa drinks associated with a transient increase in flow-mediated dilation, indicating improved endothelial function [29].

In summary, cooking a hamburger with a polyphenol-rich spice mixture may lead to potential cardiovascular benefits in patients with Type 2 diabetes mellitus.

\section{Acknowledgments}

Funding sources

DH is a member of the McCormick Science Institute and has received honoraria and travel reimbursements from the McCormick Science Institute.

\section{References}

1. Viuda-Martos M, Ruiz-Navajas Y, Fernandez-Lopez J, Perez-Alvarez JA. Spices as functional foods. Crit Rev Food Sci Nutr 2011; 51: 13-28. [PubMed: 21229415]

2. Ross R The pathogenesis of atherosclerosis: a perspective for the 1990s. Nature 1993; 362: 801809. [PubMed: 8479518]

3. Schini-Kerth VB, Auger C, Kim JH, Etienne-Selloum N, Chataigneau T. Nutritional improvement of the endothelial control of vascular tone by polyphenols: role of NO and EDHF. Pflugers Arch 2010; 459: 853-862. [PubMed: 20224869]

4. Li Z, Henning SM, Zhang Y, Zerlin A, Li L, Gao K et al. Antioxidant-rich spice added to hamburger meat during cooking results in reduced meat, plasma, and urine malondialdehyde concentrations. Am J Clin Nutr 2010; 91: 1180-1184. [PubMed: 20335545]

5. Bell DS, O'Keefe JH, Jellinger P. Postprandial dysmetabolism: the missing link between diabetes and cardiovascular events? Endocr Pract 2008; 14: 112-124. [PubMed: 18238751]

6. Sies H, Stahl W, Sevanian A. Nutritional, dietary and postprandial oxidative stress. J Nutr 2005; 135: 969-972. [PubMed: 15867266]

7. Staprans I, Hardman DA, Pan XM, Feingold KR. Effect of oxidized lipids in the diet on oxidized lipid levels in postprandial serum chylomicrons of diabetic patients. Diabetes Care 1999; 22: 300 306. [PubMed: 10333949]

8. Ursini F, Zamburlini A, Cazzolato G, Maiorino M, Bon GB, Sevanian A. Postprandial plasma lipid hydroperoxides: a possible link between diet and atherosclerosis. Free Radic Biol Med 1998; 25: 250-252. [PubMed: 9667503]

9. Williams MJ, Sutherland WH, McCormick MP, de Jong SA, Walker RJ, Wilkins GT. Impaired endothelial function following a meal rich in used cooking fat. J Am Coll Cardiol 1999; 33: 10501055. [PubMed: 10091835]

10. Phillips LK, Peake JM, Zhang X, Hickman IJ, Kolade O, Sacre JW et al. The effect of a high-fat meal on postprandial arterial stiffness in men with obesity and type 2 diabetes. J Clin Endocrinol Metab 2010; 95: 4455-4459. [PubMed: 20610595] 
11. Esposito K, Nappo F, Giugliano F, Di PC, Ciotola M, Barbieri M et al. Meal modulation of circulating interleukin 18 and adiponectin concentrations in healthy subjects and in patients with type 2 diabetes mellitus. Am J Clin Nutr 2003; 78: 1135-1140. [PubMed: 14668275]

12. Anderson RA, Evans LM, Ellis GR, Khan N, Morris K, Jackson SK et al. Prolonged deterioration of endothelial dysfunction in response to postprandial lipaemia is attenuated by vitamin C in Type 2 diabetes. Diabet Med 2006; 23: 258-264. [PubMed: 16492208]

13. Grotto D, Santa Maria LD, Boeira S, Valentini J, Charao MF, Moro AM et al. Rapid quantification of malondialdehyde in plasma by high performance liquid chromatography-visible detection. $\mathrm{J}$ Pharm Biomed Anal 2007; 43: 619-624. [PubMed: 16949242]

14. Hamburg NM, Keyes MJ, Larson MG, Vasan RS, Schnabel R, Pryde MM et al. Cross-sectional relations of digital vascular function to cardiovascular risk factors in the Framingham Heart Study. Circulation 2008; 117: 2467-2474. [PubMed: 18458169]

15. Bonetti PO, Barsness GW, Keelan PC, Schnell TI, Pumper GM, Kuvin JT et al. Enhanced external counterpulsation improves endothelial function in patients with symptomatic coronary artery disease. J Am Coll Cardiol 2003; 41: 1761-1768. [PubMed: 12767662]

16. Rice-Evans CA, Miller NJ. Antioxidant activities of flavonoids as bioactive components of food. Biochem Soc Trans 1996; 24: 790795.

17. Jovanovic SV, Simic MG. Antioxidants in nutrition. Ann N Y Acad Set 2000; 899: 326-334.

18. Kanner J, Gorelik S, Roman S, Kohen R. Protection by polyphenols of postprandial human plasma and low-density lipoprotein modification: the stomach as a bioreactor. J Agric Food Chem 2012; 60: 8790-8796. [PubMed: 22530973]

19. Lo CY, Li S, Tan D, Pan MH, Sang S, Ho CT. Trapping reactions of reactive carbonyl species with tea polyphenols in simulated physiological conditions. Mol Nutr Food Res 2006; 50: 1118-1128. [PubMed: 17103374]

20. Totlani VM, Peterson DG. Epicatechin carbonyl-trapping reactions in aqueous maillard systems: identification and structural elucidation. J Agric Food Chem 2006; 54: 7311-7318. [PubMed: 16968099]

21. Piche LA, Cole PD, Hadley M, van den BR, Draper HH. Identification of $N$-epsilon-(2propenal)lysine as the main form of malondialdehyde in food digesta. Carcinogenesis 1988; 9: 473-477. [PubMed: 3125998]

22. Giron-Calle J, Alaiz M, Millan F, Ruiz-Gutierrez V, Vioque E. Bound malondialdehyde in foods: bioavailability of $N, N$-di-(4-methyl-1,4-dihydropyridine-3,5-dicarbaldehyde)lysine. J Agric Food Chem 2003; 51: 4799-4803. [PubMed: 14705915]

23. Gee JM, DuPont MS, Rhodes MJ, Johnson IT. Quercetin glucosides interact with the intestinal glucose transport pathway. Free Radic Biol Med 1998; 25: 19-25. [PubMed: 9655517]

24. Kandra L, Gyemant G, Zajacz A, Batta G. Inhibitory effects of tannin on human salivary alphaamylase. Biochem Biophys Res Commun 2004; 319: 1265-1271. [PubMed: 15194503]

25. McDougall GJ, Stewart D. The inhibitory effects of berry polyphenols on digestive enzymes. Biofactors 2005; 23: 189-195. [PubMed: 16498205]

26. Kanner J Dietary advanced lipid oxidation endproducts are risk factors to human health. Mol Nutr Food Res 2007; 51: 1094-1101. [PubMed: 17854006]

27. Yang G, Lucas R, Caldwell R, Yao L, Romero MJ, Caldwell RW. Novel mechanisms of endothelial dysfunction in diabetes. J Cardiovasc Dis Res 2010; 1: 59-63. [PubMed: 20877687]

28. Auger C, Chaabi M, Anselm E, Lobstein A, Schini-Kerth VB. The red wine extract-induced activation of endothelial nitric oxide synthase is mediated by a great variety of polyphenolic compounds. Mol Nutr Food Res 2010; 54: S171-S183. [PubMed: 20440695]

29. Schewe T, Steffen Y, Sies H. How do dietary flavanols improve vascular function? A position paper. Arch Biochem Biophys 2008; 476: 102-106. [PubMed: 18358827] 

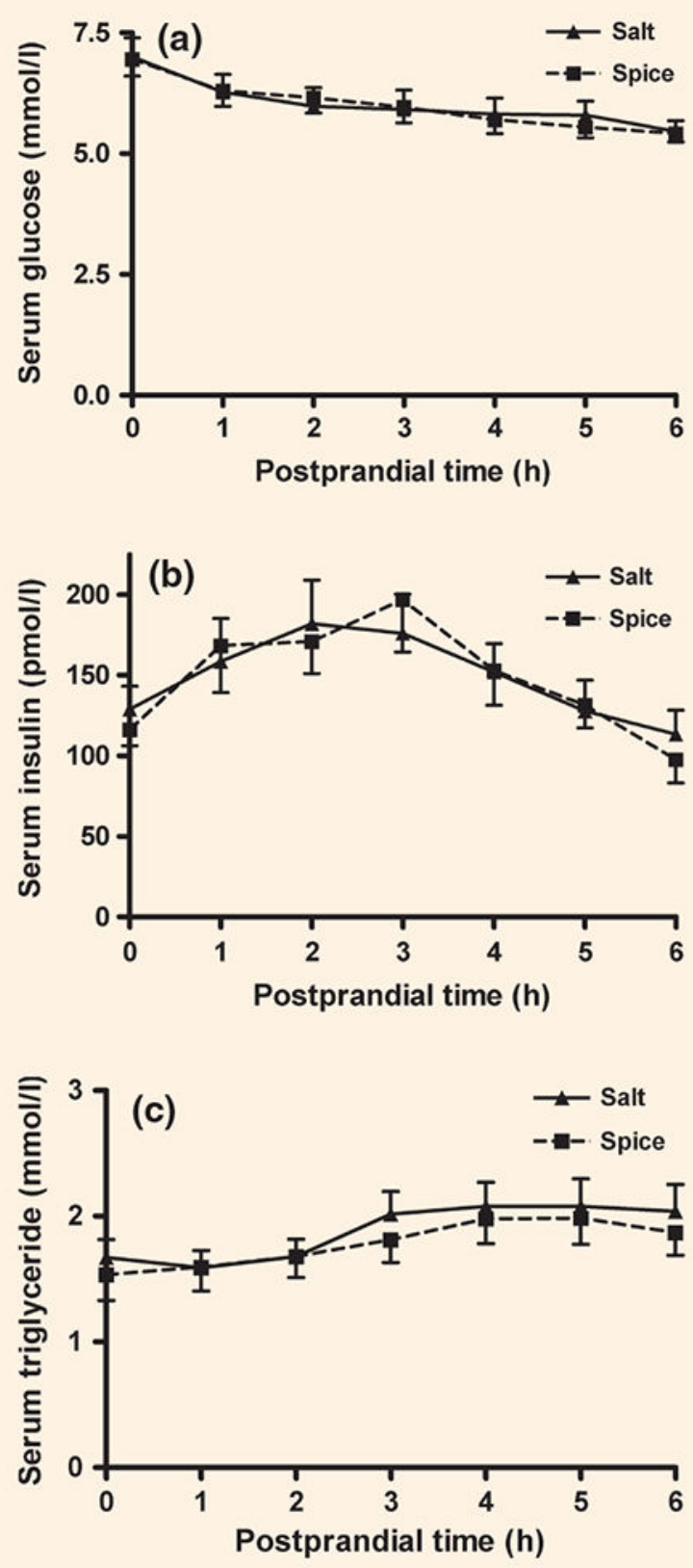

FIGURE 1.

Postprandial changes in serum glucose (a), insulin (b) and triglycerides (c) after eating a burger with salt $(\boldsymbol{\Delta})$ or spice $(\boldsymbol{\square})$. Data are mean \pm standard error of the mean (SEM). $P$-value: no significant differences were observed; $n=18$. 


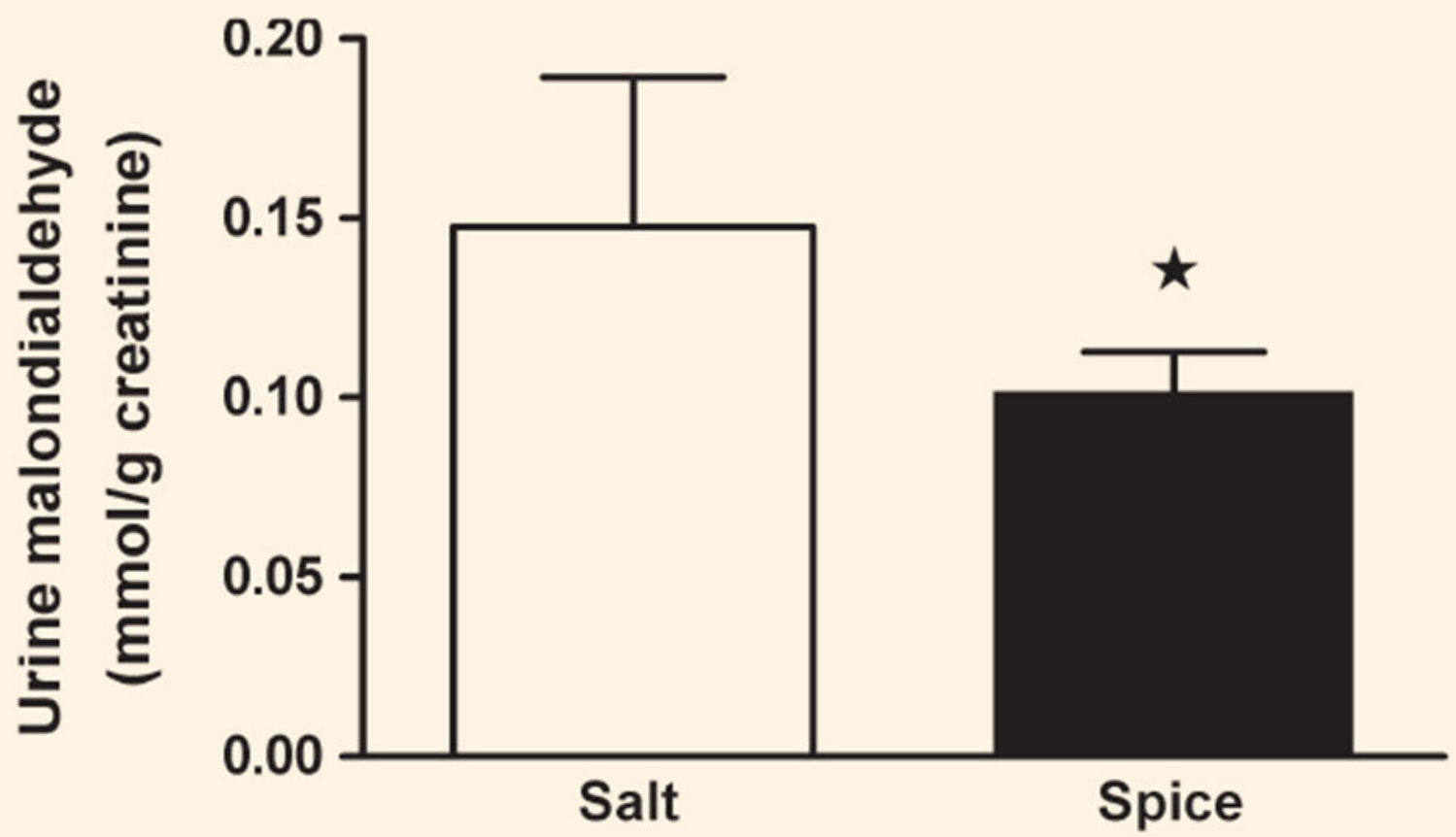

FIGURE 2.

Urinary excretion of malondialdehyde. Malondialdehyde was calculated on the basis of creatinine concentration in urine collected for $6 \mathrm{~h}$ after eating a burger with salt $(\square)$ or spice (ם). $* P<0.05$; data are mean \pm standard deviation (SD); $n=8$. 


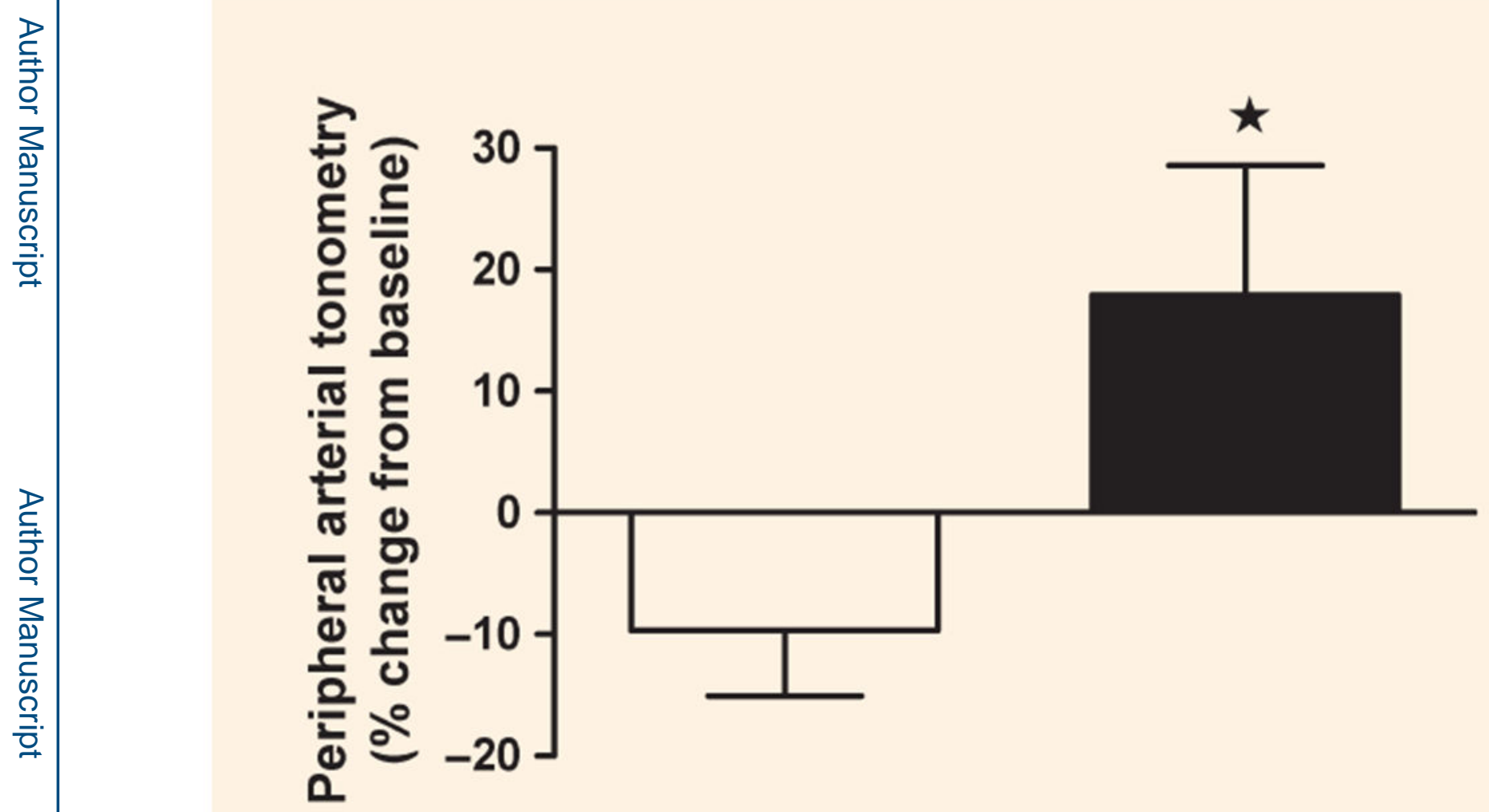

FIGURE 3.

Two-hour postprandial peripheral arterial tonometry score change from baseline after eating a burger with salt. $(\square)$ or spice ( $\square) . * P<0.05$; data are mean \pm standard deviation (SD); $n=$ 18. 


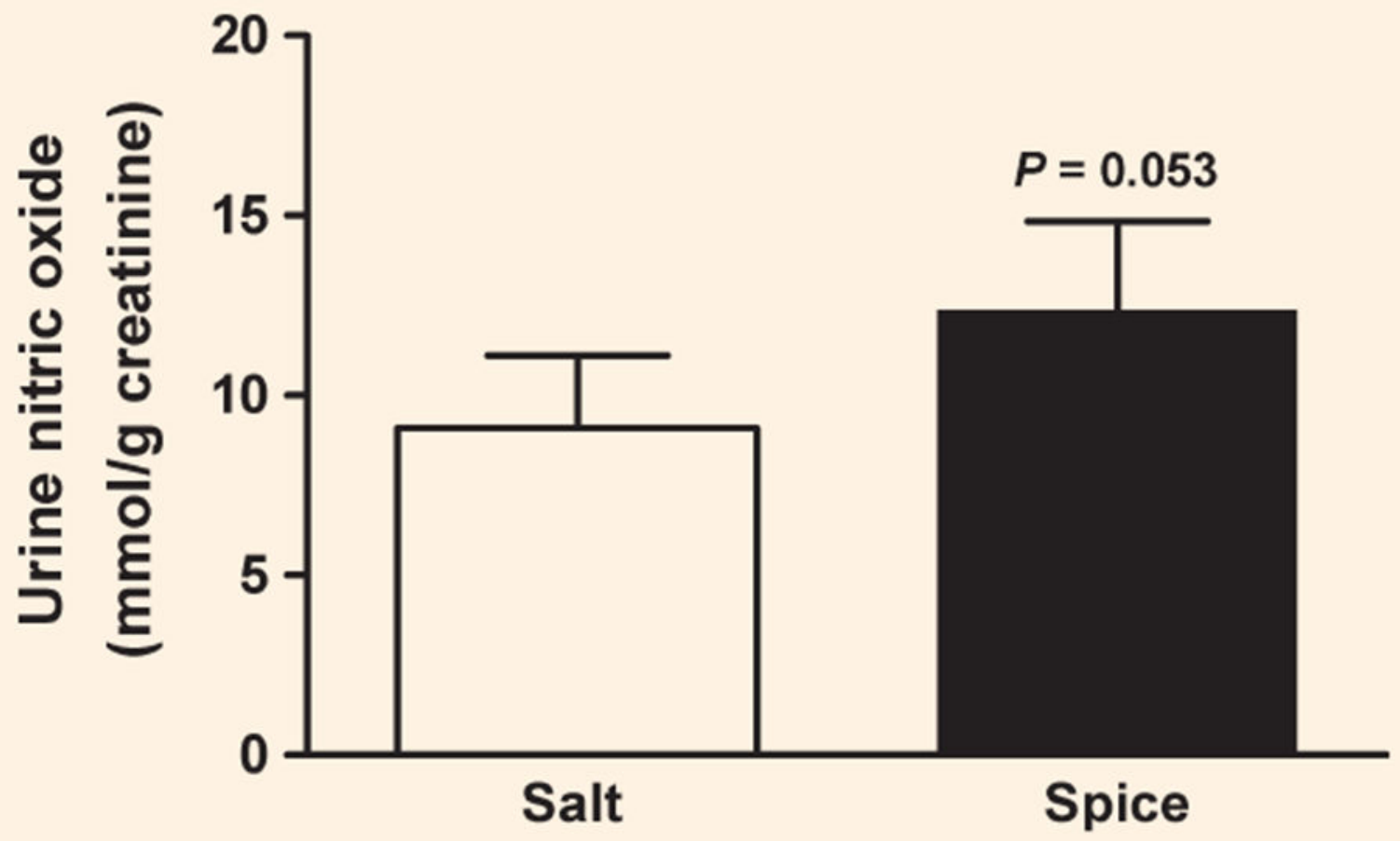

FIGURE 4.

Urine nitric oxide (NO) concentration, determined as the sum of urinary nitrate and nitrite, after eating a burger with salt $(\square)$ or spice (匹). $P=0.053$; data are mean \pm standard deviation (SD); $n=8$. 


\section{Table 1}

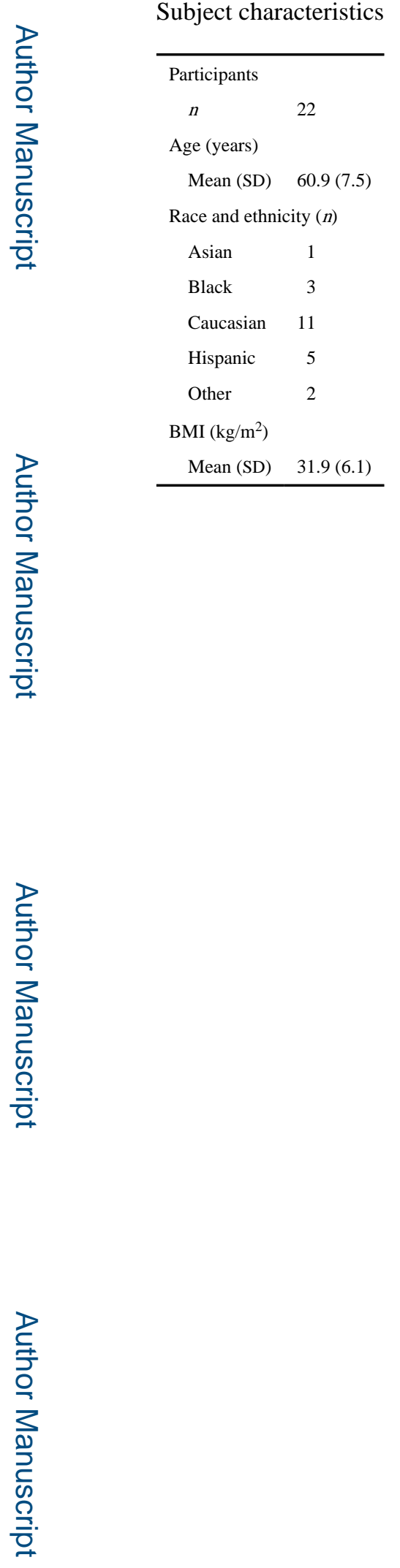

Diabet Med. Author manuscript; available in PMC 2020 July 13. 


\section{Table 2}

Concomitant medication $^{*+}$

\begin{tabular}{lc}
\hline & Number of participants taking the medication (total $\boldsymbol{n}=\mathbf{1 8})$ \\
\hline Type 2 diabetes - metformin & 13 \\
Other Type 2 diabetes medication (glypizide, glyburide and pioglitazone) & 3 \\
Hypertension (atenolol, zestril, benazepril) & 12 \\
Lipid lowering (rosuvastatin, lovestatin, simvastatin and niacin) & 9 \\
Other (antidepressant, benign prostatic hyperplasia, gastric reflux) & 8 \\
\hline
\end{tabular}

*

* All medication was prescribed by primary care physicians.

For subjects who routinely took metformin in the morning, the usual dose of metformin was given just before the consumption of the test hamburger and all other diabetes medications were withheld on the test day. 